MINI-SYMPOSIUM

\title{
Hibernation and heart failure
}

P G Camici

Heart 2004;90:141-143. doi: 10.1136/hrt.2003.023119

$\mathrm{H}$ eart failure accounts for approximately $20 \%$ of all hospital admissions among people over 65 , and in the past 10 years the hospitalisation rate has increased by almost 160\%.' Although different large randomised trials carried out during the past two decades have demonstrated a significant reduction in mortality for heart failure patients treated medically, symptomatic heart failure continues to have a one year mortality close to $45 \% .^{23}$

In an effort to improve the early recognition of heart failure, the American College of Cardiology and the American Heart Association have recently proposed a new approach to the classification of heart failure based on four progressive stages. ${ }^{4}$ This new categorisation emphasises the evolution and progression of heart failure and is quite different from the traditional New York Heart Association (NYHA) classification whose primary objective was to describe functional limitations. Although this recent classification underscores the risk factors and structural abnormalities, which are necessary for the development of heart failure, patients are considered independent of the origin of their condition and no major emphasis is placed on the aetiology.

\section{HEART FAILURE IN PATIENTS WITH CORONARY ARTERY DISEASE}

In over two thirds of cases heart failure is secondary to coronary artery disease. The increasingly successful treatment and reduced mortality of acute coronary syndromes has increased the prevalence of chronic heart failure in patients with coronary artery disease. ${ }^{5}$ Evidence from non-randomised studies suggests that in patients with post-ischaemic heart failure, coronary revascularisation can lead to symptomatic and prognostic benefits. ${ }^{5}$ To understand the reasons for the beneficial effects of revascularisation, it is necessary to review the underlying mechanisms of heart failure in patients with coronary artery disease. This is generally the result of three factors:

- Permanent myocyte loss due to infarction with scar formationThe size of the infarct can be reduced by prompt thrombolysis with rapid "door to needle" times and primary percutaneous interventions, but a degree of necrosis is usually inevitable, even if clinically silent.

- Chronic dysfunction in viable myocardium subtended by stenosed coronary arteries which recovers after revascularisation (hibernating myocardium)-This represents the myocardium where the ischaemic insult is insufficient to induce necrosis, although the myocardium remains jeopardised, prone to repeated episodes of ischaemia, and exhibits notably reduced contraction. The concept of myocardial hibernation arose from the observation that sustained improvement in left ventricular function may occur after coronary artery bypass grafting ${ }^{6}$ and that transient improvement in chronically dysfunctional segments may occur in response to inotropic stimulation. ${ }^{7}$

- Changes in the remote myocardium (adverse remodelling)-The workload of myocardium remote from the site of infarction is increased to compensate for the loss of contraction in the infarcted and hibernating segments. The changes in ventricular geometry, local wall strain, and filling pressures combine to increase the metabolic requirements of such myocardium which, while "remote" from the site of infarction, adopts a "crucial" role in maintaining cardiac output. These segments undergo compensatory hypertrophy, but in the long term adverse "remodelling" and ventricular dilatation occurs leading to heart failure. ${ }^{1}$

Based on available studies, it is logical to assume that the beneficial effect of coronary revascularisation in heart failure derives primarily from recovery of contractile function in hibernating segments which, in turn, may attenuate remodelling. ${ }^{8}$

\section{VENTRICULAR DYSFUNCTION IN HIBERNATING MYOCARDIUM AND ITS INCIDENCE}

The dysfunction associated with hibernating myocardium can be limited to a discrete portion of the left ventricle, with preserved global ejection fraction, or may be generalised and result in global impairment of ventricular function and heart failure. The severity of functional impairment can vary from hypokinesia to akinesia or dyskinesia. ${ }^{5}$

The prevalence of hibernating myocardium can be estimated from the frequency of functional recovery of regional wall motion abnormalities after revascularisation. In a prospective study of 252 patients without previous infarction who were referred for coronary angiography, evidence of hibernating myocardium was present in up to one third of all patients and $85 \%$ of the asynergic myocardial segments were found to improve their function after revascularisation. ${ }^{9}$ In patients with previous infarction, areas of hibernating tissue may be intermixed with scar in up to $50 \%$ of the cases, even in the presence of Q waves on the ECG. ${ }^{10}$ The incidence and degree of functional recovery following coronary revascularisation depends on a number of factors such as patient selection, techniques for myocardial protection, the occurrence of perioperative myocardial infarction, and adequacy of revascularisation. From the available literature regional functional recovery varies widely from $24-82 \%$ of all segments. ${ }^{5}$ It follows that at least a quarter of all the patients with proven coronary artery disease have some degree of hibernating myocardium.

\section{IDENTIFICATION OF PATIENTS WITH HIBERNATING MYOCARDIUM}

In principle, hibernating myocardium should be suspected in all patients with coronary artery disease and chronic left ventricular dysfunction of any degree ranging from regional dysfunction to ischaemic cardiomyopathy. Other causes of left ventricular dysfunction, such as ethanol abuse and tachycardiomyopathy, that can be associated with coronary

Abbreviations: MBF, myocardial blood flow; NYHA, New York Heart Association; PET, positron emission tomography; SPECT, single photon emission computed tomography 
artery disease, should be excluded. If the demonstration of coronary artery disease is the condition sine qua non for the definition of hibernation, the presence and severity of left ventricular dysfunction is not necessarily commensurate to the extent and severity of coronary artery disease. In many patients the recruitment and development of coronary collaterals can result in the preservation of normal left ventricular function despite the presence of sometimes severe coronary artery disease. ${ }^{11}$

Myocardial hibernation implies the concept of tissue viability, which can be diagnosed by imaging modalities that detect either the presence of contraction reserve or the persistence of metabolic activity within chronically dysfunctional myocardial regions (see Kim and Shah on page 137).

\section{PATHOPHYSIOLOGY OF MYOCARDIAL HIBERNATION}

The debate on whether resting myocardial blood flow (MBF) to hibernating myocardium is reduced or not has attracted a lot of interest and, undoubtedly, has stimulated new research on heart failure in patients with coronary artery disease. Although the debate is not yet over, some of the initial paradigms have been proven incorrect while new pathophysiological concepts have emerged.

The initial hypothesis that hibernation is caused by a down regulation of myocardial function, secondary to a reduction of resting blood flow beyond a flow limiting stenosis, ${ }^{6}$ was supported by a series of studies in which semiquantitative measurements of MBF were performed using different radioactive flow tracers with single photon emission computed tomography (SPECT). ${ }^{12}$ Although radionuclide imaging techniques like thallium-201 SPECT enable the assessment of nutritive tissue perfusion, they can only provide images that reflect relative regional radioactivity concentration rather than enabling measurement of absolute MBF. ${ }^{12}$

More recently, a number of studies using positron emission tomography (PET) have demonstrated that MBF in patients with hibernating myocardium segments is generally within the range of values seen in healthy volunteers. ${ }^{12}$ PET overcomes the physical limitations of SPECT, thus enabling accurate quantification of the concentration of radiolabelled tracer in the organ of interest and allows the non-invasive measurement of $\mathrm{MBF}$ in humans $(\mathrm{ml} / \mathrm{min} / \mathrm{g})$.

The literature can, on occasion, appear confused, as variable results have been reported in different PET studies on resting $\mathrm{MBF}$ in patients with hibernating myocardium. ${ }^{12}$ There are a number of technical reasons that affect the various PET studies to varying extents and can explain, at least in part, this apparent discrepancy. In nine out of 15 studies-in whom hibernation was correctly defined as evidence of functional recovery after revascularisation in a previously dysfunctional segment subtended by a stenotic artery-flow in hibernating segments was not significantly different from that in remote, normally contracting myocardium. In the remaining six studies, flow in hibernating segments was $\sim 20 \%$ lower compared to remote myocardium. Although the paired comparison of flow in dysfunctional and remote areas is statistically more powerful than comparing patients with a normal matched population, the data need to be carefully weighed and regional differences in cardiac workload considered. The difference observed might be explained, at least in part, by a higher MBF in the remote normally contracting regions rather than by an absolute reduction in hibernating segments. The latter would be consistent with the higher oxygen consumption reported in regions remote from segments with severe wall motion abnormalities.

\section{CORONARY FLOW RESERVE AND HIBERNATION}

The coronary flow reserve is the ratio of MBF during near maximal vasodilatation (pharmacologically induced) to resting blood flow and is an index of the functional significance of coronary stenoses. In patients with coronary artery disease, flow reserve decreases in proportion to the degree of stenosis severity and is abolished (that is, hyperaemic flow = resting flow) for stenoses $\geqslant 80 \%$ of the luminal diameter. ${ }^{13}$ Under these circumstances, any increase in cardiac workload above baseline conditions cannot be met by an adequate increase in MBF, leading to ischaemia. Therefore, in patients with severe coronary artery disease the limited flow reserve leads to the development of myocardial ischaemia, which is often asymptomatic, even for small increases of oxygen demand such as those associated with ordinary daily activities. Regardless of the blood flow level under baseline conditions, these patients will develop ischaemia when oxygen demand is increased (demand ischaemia). Ischaemia is invariably associated with the development of post-ischaemic contractile dysfunction that persists following reperfusion, despite the restoration of normal or near normal coronary blood flow. In the mid 1970s this phenomenon, later known as myocardial stunning, was initially described by Heyndrickx and colleagues ${ }^{14}$ as a sustained, but eventually completely reversible, post-ischaemic contractile dysfunction in a conscious healthy dog model subjected to a 15 minute coronary occlusion.

Two decades later it was shown that patients with coronary artery disease and absence of contractile dysfunction at baseline may also develop myocardial stunning after induction of ischaemia with exercise or dobutamine. ${ }^{15}$ In addition, we have recently demonstrated that repeated episodes of ischaemia may have a cumulative effect and culminate in more prolonged and severe post-ischaemic stunning. ${ }^{17}$

In the long term, intermittent episodes of ischaemia followed by stunning might induce regional alterations in the myocytes thus contributing to the development of persistent, but still reversible, left ventricular dysfunction similar to that observed in hibernation. Clearly, under these conditions, coronary revascularisation, by restoring flow reserve, could interrupt the vicious circle that has led to this chronic post-ischaemic dysfunction.

\section{CONCLUSION}

A more correct and inclusive definition of hibernating myocardium must encompass chronically stunned myocardium. The unifying feature of this entity is reduced coronary flow reserve caused by coronary artery disease. Considering the underlying pathophysiology and the ubiquity of stress induced ischaemia in patients with coronary artery disease, it is important to recognise the clinical prevalence of hibernating myocardium and how therapeutic interventions can result in improved patient care and prognosis.

Correspondence to: Paolo G Camici MD, MRC Clinical Sciences Centre, Hammersmith Hospital, Ducane Road, London W12 ONN, UK ; paolo.camici@csc.mrc.ac.uk

\section{REFERENCES}

1 Jessup M, Brozena S. Heart failure. N Engl J Med 2003;348:2007-18.

2 Khand A Gemmel I, Clark AL, et al. Is the prognosis of heart failure improving? J Am Coll Cardiol 2000;36:2284-6.

3 Konstam MA. Progress in heart failure management? Lessons from the real world. Circulation 2000;102:1076-8.

4 Hunt SA, Baker DW, Chin MH, et al. ACC/AHA guidelines for the evaluation and management of chronic heart failure in the adult: executive summary. A report of the American College of Cardiology/American Heart Association task force on practice guidelines (committee to revise the 1995 guidelines for the evaluation and management of heart failure). J Am Coll Cardiol $2001 ; 38: 2101-13$. 
5 Wiins W, Vatner SF, Camici PG. Hibernating myocardium. N Engl J Med 1998;339:173-81.

6 Rahimtoola SH. A perspective on the three large multicenter randomized clinical trials of coronary bypass surgery for chronic stable angina. Circulation $1985 \cdot 72(6$ Pt 2):V123-35.

7 Helfant RH, Pine R, Meister SG, et al. Nitroglycerin to unmask reversible asynergy. Correlation with post coronary bypass ventriculography. Circulation 1974;50:108-13.

8 Hamer AW, Takayama M, Abraham KA, et al. End-systolic volume and longterm survival after coronary artery bypass graft surgery in patients with impaired left ventricular function. Circulation 1994;90:2899-904.

9 Lewis SJ, Sawada SG, Ryan T, et al. Segmental wall motion abnormalities in the absence of clinically documented myocardial infarction: clinical significance and evidence of hibernating myocardium. Am Heart $J$ 1991;121(4 Pt 1):1088-94.

10 Brunken R, Tillisch J, Schwaiger $M$, et al. Regional perfusion, glucose metabolism, and wall motion in patients with chronic electrocardiographic $Q$ wave infarctions: evidence for persistence of viable tissue in some infarct regions by positron emission tomography. Circulation 1986;73:951-63.

11 Vanoverschelde JL, Wijns W, Depre C, et al. Mechanisms of chronic regional postischemic dysfunction in humans. New insights from the study of noninfarcted collateral-dependent myocardium. Circulation 1993;87:1513-23.

12 Camici PG, Rimoldi OE. Myocardial blood flow in patients with hibernating myocardium. Cardiovasc Res 2003;57:302-11.

13 Uren NG, Melin JA, De Bruyne B, et al. Relation between myocardial blood flow and the severity of coronary-artery stenosis. N Engl J Med 1994;330:1782-8.

14 Heyndrickx GR, Millard RW, McRitchie RJ, et al. Regional myocardial functional and electrophysiological alterations after brief coronary artery occlusion in conscious dogs. J Clin Invest 1975;56:978-85.

15 Ambrosio G, Betocchi S, Pace L, et al. Prolonged impairment of regional contractile function after resolution of exercise-induced angina. Evidence of myocardial stunning in patients with coronary artery disease. Circulation 1996;94:2455-64.

16 Barnes E, Hall RJ, Dutka DP, et al. Absolute blood flow and oxygen consumption in stunned myocardium in patients with coronary artery disease. J Am Coll Cardiol 2002;39:420-7.

17 Barnes $E$, Dutka DP, Khan $M$, et al. Effect of repeated episodes of reversible myocardial ischemia on myocardial blood flow and function in humans. Am J Physiol Heart Circ Physiol 2002;282:H1603-8.

\section{IMAGES IN CARDIOLOGY}

\section{Infectious endocarditis with an abscess around an aortic prosthesis}

A 50 year old man was admitted for deterioration in his general condition, with a temperature of $38.7^{\circ} \mathrm{C}$, shivering and asthenia. His history included idiopathic cirrhosis of the liver with portal hypertension and type 2 diabetes. Eighteen months previously, his aortic valve had been replaced with a Carpentier-Edwards bioprosthesis to correct pronounced calcific nodular aortic stenosis.

On admission the following signs were noted: crepitants in the base of the lungs; transprosthetic aortic systolic murmur; hyperleukocytosis $18.3 \times 10^{9} / 1$ (normal $<11$ ); thrombocytopenia $75 \times 10^{9} / 1$ (normal $\left.>160\right)$; C reactive protein $145 \mathrm{mg} / \mathrm{l}$ (normal <10); and negative blood cultures. Transoesophageal echocardiography revealed moderate aortic insufficiency secondary to partial displacement of the bioprosthesis, a circulating abscess with systolic expansion around the aorto-mitral trigonum (below left), and a vegetation attached to the aortic bioprosthesis (below right).

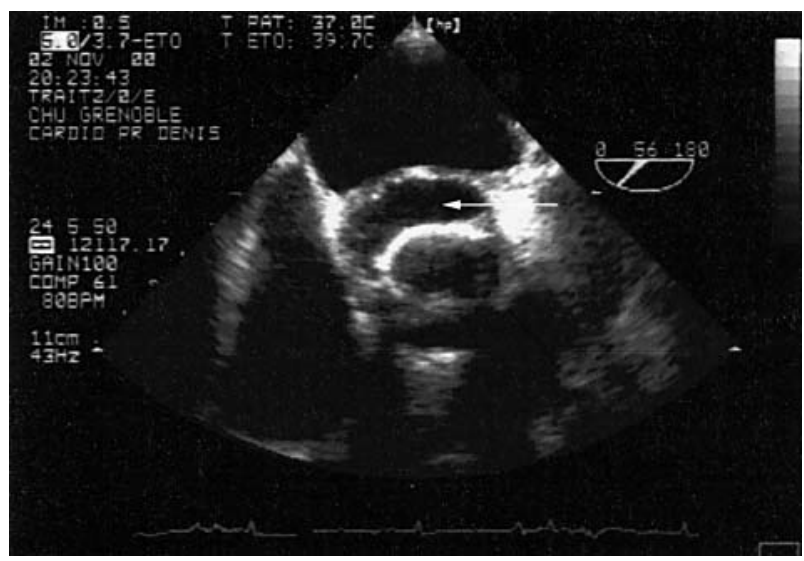

Transoesophageal echocardiography showing a circulating abscess (arrow) around the Carpentier-Edwards aortic bioprosthesis.
On commencement of antibiotic treatment (amoxicillin plus netilmicin), the patient's general condition improved and the signs of inflammation waned. Five days later, the patient presented with acute pulmonary oedema and suffered cardiac arrest. Transthoracic ultrasound revealed massive aortic insufficiency and emergency surgery was performed. The aortic prosthesis was found to have almost completely come out so a Carbomedics model had to be implanted. The patient's condition subsequently deteriorated with progressive multi-organ failure, and he died 16 days after the operation.

J-P Baguet

O Chavanon

M-A Lebrun

JPBaguet@chu-grenoble.fr

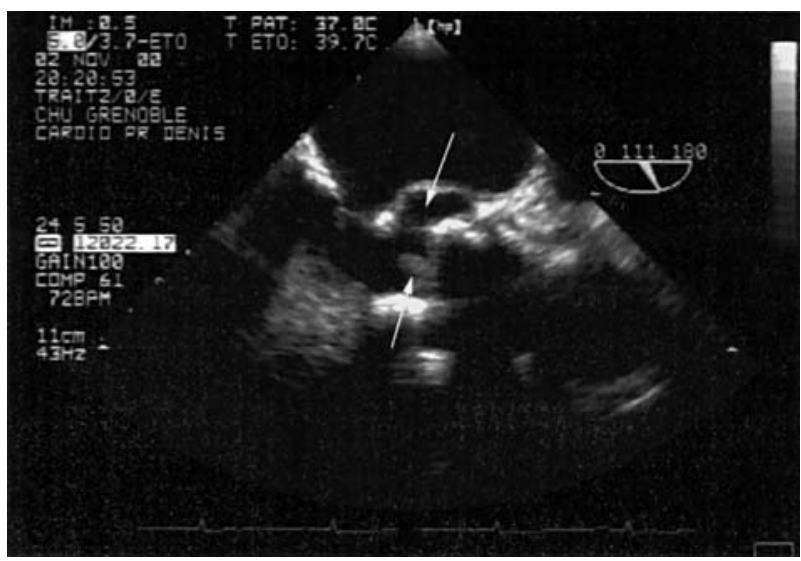

Transoesophageal echocardiography showing the communication between an abscess of the aorto-mitral trigonum and the ejection chamber of the left ventricle together with a vegetation attached to a cusp of the Carpentier-Edwards aortic bioprosthesis. 